\title{
The Diurnal Cortisol Cycle in Delinquent Male Adolescents and Normal Controls
}

\author{
Arne Popma*,', Theo AH Doreleijers', Lucres MC Jansen', Stephanie HM Van Goozen², \\ Herman Van Engeland ${ }^{3}$ and Robert Vermeiren' \\ 'Department of Child and Adolescent Psychiatry, VU University Medical Center, Amsterdam, The Netherlands; ${ }^{2}$ University Medical Centre \\ Utrecht, Utrecht, The Netherlands; ${ }^{3}$ Department of Child and Adolescent Psychiatry, VU University Medical Center, Utrecht, The Netherlands
}

\begin{abstract}
Patterns of low hypothalamus-pituitary-adrenal (HPA) activity have been observed in antisocial groups. As conflicting results have been reported in children and adolescents, the aim of this study was to further investigate HPA activity in antisocial behavior by studying the relationship between the diurnal cortisol cycle, as well as the cortisol awakening response (CAR), and antisocial behavior in male adolescents. The diurnal cortisol cycle and the CAR during the first hour after awakening were compared between 12-to I4-year-old boys who attended a delinquency diversion program (DP), with and without a disruptive behavior disorder (DBD) (respectively DP + ; $n=24$ and DP-; $n=65)$, and matched normal controls $(N C ; n=32)$. The DP + group, but not the DP- group, showed a significantly slower decrease of cortisol during the diurnal cycle than the NC group. Furthermore, the DP + group had significantly lower cortisol levels in the first hour after awakening as compared with the NC group. The results indicate altered HPA activity in delinquent boys with a DBD. Etiological mechanisms, directions for future research, and clinical implications are discussed.

Neuropsychopharmacology (2007) 32, 1622-1628; doi: I0.1038/sj.npp. I 30 1289; published online 17 January 2007
\end{abstract}

Keywords: cortisol; HPA axis; cortisol awakening response; delinquency; disruptive behavior; conduct disorder

\section{INTRODUCTION}

There is evidence of an association between low hypothalamus-pituitary-adrenal (HPA) axis activity and antisocial behavior. Although these findings are quite robust in adults (Virkkunen, 1985), findings in children and adolescents have been less consistent (Van Goozen et al, 2000). Notwithstanding the value of the existing studies in minors, methodological limitations must be recognized, for example, not taking into account the circadian rhythm of the HPA axis. Therefore, this study will focus on the association between the entire diurnal cycle of cortisol, as well as the cortisol awakening response (CAR), and antisocial behavior, in a group of 12- to 14-year-old male adolescents attending a delinquency diversion program (DP) and in a matched normal control group.

Antisocial behavior by children is a major public health problem, with disruptive behavior problem children costing society at least 10 times as much as well developing children (Scott et al, 2001). Antisocial children are at risk for a series of negative outcomes in adulthood, such as criminal

*Correspondence: Dr A Popma, Department of Child and Adolescent Psychiatry, VU University Medical Center, Amsterdam, P/a De Bascule, Rijksstraatweg I45, PB 303, I I 5 ZG Duivendrecht, The Netherlands, Tel: +31208901545, Fax: + 31206952541 ,

E-mail: a.popma@debascule.com

Received 26 April 2006; revised 6 September 2006; accepted II October 2006 behavior, social isolation, unemployment, and psychiatric disorders, including depression, anxiety disorders, and substance abuse (Maughan and Rutter, 2001). Neurobiological mechanisms may be important in explaining individual variation in antisocial behavior. There is increasing evidence that children with disruptive behavior problems, and particularly those who show persistent antisocial behavior, are characterized by neurobiological deficits (Raine, 2002). In this respect, stress regulating mechanisms, for example, the HPA axis, have drawn much attention (Kruesi et al, 1989; Raine, 2002; Vanyukov et al, 1993; Kerr et al, 1997; Mezzacappa et al, 1997). A primary focus in this field of research has been cortisol, which is the final product of the HPA axis and the primary stress hormone in humans. As cortisol is considered a parameter of arousal, it has been studied within the paradigm of the low arousal theory. This theory postulates low levels of arousal to be associated with high levels of antisocial behavior, which has been explained predominantly in two ways. First, the fearlessness theory claims that individuals with low arousal levels are more likely to engage in delinquent acts, physical fights and other forms of antisocial behavior because they do not fear the negative consequences of their aggressive actions (Raine, 1993). A second interpretation is provided by the sensationseeking theory (Zuckerman, 1979), in which it is argued that low arousal levels represent an aversive physiological state. As such, individuals with low arousal levels are motivated to seek out stimulation in order to raise their arousal levels to 
an optimal or normal level. In this view, engaging in antisocial behavior constitutes a way of seeking stimuli.

While an association between low cortisol levels and antisocial behavior has consistently been found in adults (Virkkunen, 1985; King et al, 2001; Woodman et al, 1978), findings in children and adolescents are less clear. Some studies in minors demonstrated low cortisol levels in resting conditions to be associated with antisocial behavior (Pajer et al, 2001; McBurnett et al, 2000; Tennes et al, 1986; Tennes and Kreye, 1985), whereas others did not report a significant relationship (Azar et al, 2004; Schulz et al, 1997; Kruesi et al, 1989; Scerbo and Kolko, 1994; Klimes-Dougan et al, 2001; Stoff et al, 1992). In a recent longitudinal study, low cortisol levels in preadolescence were found to be associated with low harm avoidance, low self-control, and more aggressive behavior when reaching middle adolescence 5 years later (Shoal et al, 2003).

Only a few studies in behavior disordered children have measured cortisol repeatedly on one day, mostly during various experimental conditions. Although no differences in baseline cortisol levels were reported in these studies, lower cortisol levels during stress were found in children with a disruptive behavior disorder (DBD) compared with normal controls (NC) (Van Goozen et al, 2000, 1998; Moss et al, 1995). In contrast, a positive relationship was found in healthy adolescents between antisocial behavior and cortisol levels during experimentally induced aggression (Gerra et al, 1997) and during an emotion arousing and a painful procedure (Susman et al, 1997).

Despite the great value of prior studies in minors, methodological issues may underlie some of the inconsistencies found. First, a large number of measures of antisocial behavior and aggression have been investigated, ranging from various self-report questionnaires on aggression to psychiatric diagnoses like DBD (Van Goozen et al, 2000). As such, thus far it remains unclear whether low cortisol is characteristic of antisocial behavior in general or is more specifically observed in those cases with a DBD diagnosis. Moreover, even DBD groups have been defined in various ways, for example, including attention deficit hyperactivity disorder (ADHD) comorbidity or not. Results from a recent study suggest though, that low HPA activity is a specific marker for DBD but not for ADHD (Snoek et al, 2004).

Second, other methodological issues that may underlie inconsistencies in the literature, have to do with the sampling of cortisol, having been collected from plasma, urine, and saliva. Currently, sampling cortisol from saliva is the preferred method, with salivary cortisol levels accurately reflecting serum-free cortisol, which is the physiologically active component (Aardal and Holm, 1995; Aardal-Eriksson et al, 1998; Kirschbaum and Hellhammer, 1994). Moreover, this method allows cortisol to be measured repeatedly over the day in a reliable, non-intrusive, and stress-free way in ambulatory settings (Dettling et al, 1999). So far though, many studies have relied on single cortisol measurement only, with different studies sampling at different times of the day. This might have influenced results considerably, as HPA axis activity is characterized by a robust circadian rhythm. Cortisol levels peak in the early morning, gradually decline over the day, and reach its lowest levels around midnight. This diurnal cortisol cycle is an established marker of HPA activity and can be measured by sampling cortisol repeatedly during the day. The cycle represents the 'basal' secretory activity of the HPA axis (Edwards et al, 2001). Recently, a special component of this cycle, the CAR, has been proposed as distinct and reliable marker for studying HPA functioning (Pruessner et al, 1997). Within $30 \mathrm{~min}$ after awakening, mean cortisol levels increase $50-100 \%$, remaining elevated for at least $1 \mathrm{~h}$. As such, the CAR is superimposed on the underlying diurnal pattern, comprising aspects of basal activity as well as dynamic changes during the first hour after awakening (Clow et al, 2004). Several studies found no considerable impact of age, smoking habits, sleep quality, total slept time, and physical activity on the CAR (Pruessner et al, 1997; Kudielka et al, 2003). The influence of awakening time is still debated, with some studies reporting no influence (Wust et al, 2000), and others finding an association (Edwards et al, 2001). The CAR has a high intraindividual stability compared with single basal morning cortisol values (Coste et al, 1994). As such, the CAR could be an interesting parameter of additional value for studying HPA axis functioning.

Because studies on the relationship between the diurnal cycle of cortisol and antisocial behavior in children are lacking, this study aimed to investigate the diurnal cycle, as well as the CAR, in 12- to 14-year-old males referred to a delinquency DP and matched NC. As DP subjects were expected to differ with respect of amount and severity of conduct problems, and to address the issue whether altered HPA activity is specific for antisocial behavior in general or for DBD in particular, the purpose was to subdivide the DP group into two subgroups based on the presence or absence of DBD (respectively DP + and $\mathrm{DP}-$ ). It was predicted that the DP group would have lower cortisol levels than the NC group, with this effect being more apparent in the subgroup of DP boys with a DBD.

\section{MATERIALS AND METHODS}

\section{Subjects}

The sample included both adolescents attending a delinquency diversion program (DP group; $n=114$ ) in and around Amsterdam for committing a crime, and a matched group of normal controls (NC group; $n=32$ ). In the Netherlands, children between 12 and 18 years old who have committed a minor offense can be sent to a DP to prohibit court intervention. This option is only available for specific types of petty offences: wanton destructiveness, vandalism, simple theft, hooliganism, infractions on the firework regulations, and minor forms of aggression. Children in the lowest age group (12-14 years) were included between January 2002 and July 2004. This strategy was chosen in order to recruit a sample of boys with various levels of disruptive behavior problems, with a subgroup not having a $\mathrm{DBD}$, of which some subjects in the future might prove to have been a single time offender and of which others might be developing DBD, and a second subgroup displaying serious enough disruptive behavior to score a DBD diagnosis. All subjects were referred as first offenders. NC were recruited from local schools and football clubs. Delinquent boys and NC were matched group-wise for age, IQ, SES, and ethnicity (see Table 1). 
Contact with subjects and their parents was established by the research team at the offices of the participating diversion projects within 4-10 weeks after arrest by the police. Exclusion criteria for participation were a history of any neurological or endocrinological disorder, steroid medication, and IQ less than 70 .

This study was approved of by the Medical Ethical Committee of the VU University medical center of Amsterdam. The potential subjects and their parents were given a thorough outline of the procedures, were informed of their right of refusal, and were assured that refusal to participate would not influence the procedure of the diversion project. Participating parents and subjects gave written informed consent. Data collected in this study were not available to employees of the diversion project or the Juvenile Justice System.

\section{Procedure for Saliva Collection and Analysis}

Subjects sampled saliva for cortisol assessment immediately after awakening (sample 1), 30 and $60 \mathrm{~min}$ after awakening (samples 2 and 3), at 1230 (before lunch; sample 4), at 1520 and 1540 (mean = sample 5), and at 2000 and before going to bed (mean=sample 6), using the Salivette sampling device (Sarstedt, Nümbrecht, Germany). All cortisol samples were obtained on the same weekday, with all subjects spending the morning and early afternoon at school. Participants and parents were instructed both in writing

Table I Characteristics, CBCL Scores and Awakening Time of Subjects

\begin{tabular}{|c|c|c|c|}
\hline & NC $(n=32)$ & $D P-(n=65)$ & $\mathrm{DP}+(n=24)$ \\
\hline Age & $13.50 \pm 0.66$ & $13.7 \mid \pm 0.77$ & $13.42 \pm 0.56$ \\
\hline $\mathrm{IQ}$ & $97.77 \pm 10.03$ & $93.64 \pm 11.71$ & $95.16 \pm 14.15$ \\
\hline \multicolumn{4}{|l|}{ SES } \\
\hline Low & $14(43.8 \%)$ & $32(49.2 \%)$ & $10(41.7 \%)$ \\
\hline Middle & 10 (31.3\%) & $17(26.2 \%)$ & 7 (29.2\%) \\
\hline High & $8(25.0 \%)$ & $16(24.6 \%)$ & $7(29.2 \%)$ \\
\hline \multicolumn{4}{|l|}{ Ethnicity } \\
\hline Caucasian & $13(40.6 \%)$ & $26(40.0 \%)$ & $9(37.5 \%)$ \\
\hline Insurinam/antillean & $8(25.0 \%)$ & $16(24.6 \%)$ & $6(25.0 \%)$ \\
\hline Mediterrenean & $6(18.8 \%)$ & $19(29.2 \%)$ & $5(20.8 \%)$ \\
\hline Other & $5(15.6 \%)$ & $4(6.2 \%)$ & $4(26.7 \%)$ \\
\hline
\end{tabular}

\begin{tabular}{lccc} 
CBCL-scales & & & \\
Delinquency $^{\mathrm{a}}$ & $53.36 \pm 6.07$ & $57.79 \pm 7.61^{\mathrm{b}}$ & $64.90 \pm 10.10^{\mathrm{c}, \mathrm{d}}$ \\
Aggression $^{\mathrm{a}}$ & $55.89 \pm 6.89$ & $57.73 \pm 7.78$ & $64.24 \pm 10.57^{\mathrm{c}, \mathrm{d}}$ \\
& & & \\
Awakening time & $7: 23 \pm 0: 53$ & $7: 20 \pm 0: 53$ & $7: 37 \pm 0: 56$ \\
\hline
\end{tabular}

Data are presented as means and SD or number and percentage within subgroup.

CBCL, Child Behavior Checklist.

${ }^{a}$ Significant overall difference between groups.

${ }^{\mathrm{b}}$ Trend toward significant difference compared with NC $(p=0.058)$.

'Significant difference compared with NC.

${ }^{\mathrm{d}}$ Significant difference compared with DP-. and orally by a member of the research team. The day before the day of cortisol sampling, all participants were called to remind them of the sampling procedure. Subjects were instructed to write down the exact sampling times. In addition, subjects were asked not to eat between sample 1 and sample 2. They were told to rinse their mouth thoroughly with tap water before sampling saliva, not to eat fruit or to drink coffee, and not to brush their teeth before completing saliva sampling in order to avoid contamination of saliva with blood, caused by microinjuries in the oral cavity. To obtain greater compliance and increase the accuracy of reported sampling times, subjects and parents were told that accuracy of sampling times could also be monitored from the saliva samples in the lab. It has been demonstrated that subjects who know that their sampling accuracy will be checked show higher compliance than subjects who have not been informed on this (Broderick et al, 2004). Samples 1-4 were sampled at home. These were handed over to a member of the research team at 1500 when subjects and their parents arrived at the institute. Sample 5 was calculated taking the mean of two cortisol samples taken at 1520 and 1540 . Samples $1-5$ were stored in the freezer the same day. At night, all participants sampled saliva two more times at home: at 2000 and before going to bed. Sample 6 was calculated by taking the mean of these two samples. Evening samples were stored in the freezer by participants to be collected within a week by a research assistant and stored with the other samples.

All subjects with more than one missing cortisol sample were excluded $(n=17)$. Single missing values were replaced by the group average of that time point. Also, to minimize artifacts owing to differences in awakening time, subjects waking up more than $2 \mathrm{SD}$ later or earlier than the mean awakening time were excluded $(n=3)$. For samples 2 and 3, a time window of $\pm 15 \mathrm{~min}$ was allowed, whereas for other samples this was $\pm 1 \mathrm{~h}$, resulting in three exclusions. Two more subjects were excluded because of technical problems during the lab analyses. As such, of the original group of 114 DP subjects, a total of 25 subjects were left out of the current analyses. After these exclusions, awakening time did not differ significantly between subgroups (see Table 1). Also, the group of excluded subjects did not differ significantly from the group of subjects kept in the analyses with respect to age, IQ, SES, or ethnicity.

Uncentrifuged samples were stored at $-20^{\circ} \mathrm{C}$ until analysis. Salivary cortisol levels were determined in duplicate by direct radioinmmunoassay, using 125I-cortisol and antiserum made against the 3-CMO-BSA conjugate (Sulon et al, 1978). The lower detection limit of the assay was $7 \mathrm{ng} / \mathrm{dl}$, with mean intra- and inter-assay coefficients of variation of, respectively, $4.3 \%(n=10)$ and $9.4 \%(n=30)$.

\section{Instruments}

The National Institute of Mental Health (NIMH) Diagnostic Interview Schedule for Children (DISC), version IV (Shaffer et $a l, 2000)$ is an extensive semistructured psychiatric interview. The sections for assessing attention-deficit hyperactivity disorder (ADHD), oppositional defiant disorder (ODD), conduct disorder (CD), and post-traumatic stress disorder (PTSD) of both the child and parent versions of the instrument were used. Interviewers were trained for 
administering the Dutch version of the DISC. Subjects were scored having a psychiatric diagnosis based on meeting criteria from either the youth or parent report (Pajer et al, 2001). As ODD and CD are highly inter-related (Lahey et al, 1992), subjects who scored either or both of these diagnoses were classified as having a DBD. The DP group then was split into a subgroup with and without a DBD diagnosis (respectively DP $+; n=24$ and $\mathrm{DP}-; n=65$ ). Of the DP + subjects, eight had a comorbid ADHD diagnosis, whereas 10 of the DP- subjects had an ADHD diagnosis. None of the subjects had PTSD. Of the control subjects no one scored a diagnosis on the DISC.

Within the DP group, four subjects (three DP- and one $\mathrm{DP}+$ ) were on methylphenidate medication. These subjects were asked to not take their medication on the day of participation in this study, even though methylphenidate treatment has previously been shown to have no effect on cortisol levels (Weizman et al, 1987). Substance use was only reported by a minority of the subjects, probably because of the young age of participants. Over the last month before the test date, 13 subjects had smoked at least one Cigarette $(\mathrm{NC}=1, \mathrm{DP}-=5, \mathrm{DP}+=7), 23$ subjects had been drinking alcohol on at least 1 day $(\mathrm{NC}=3, \mathrm{DP}-=14$, $\mathrm{DP}+=6$ ), whereas seven subjects had been smoking cannabis on at least one day $(\mathrm{NC}=1, \mathrm{DP}-=3, \mathrm{DP}+=3)$.

To obtain dimensional data on externalizing behavior problems, the Child Behavior Checklist (Achenbach, 1991) was completed by the DISC-interviewed parent. Two subtests (Vocabulary and Block-design) of the WISC-R intelligence test (Wechsler, 1974) were used to estimate IQ.

\section{Statistical Analyses}

In order to analyze behavioral differences, CBCL T-scores of the externalizing scales were compared between groups $(\mathrm{DP}+v s \mathrm{DP}-v s \mathrm{NC})$ using one-factor analyses of variance (ANOVAs). Because cortisol values were positively skewed, they were transformed to the natural logarithm scale after which all cortisol values were normally distributed as tested with a Kolmogorov-Smirnov analysis. For reasons of physiological meaningfulness, the graphical representation of the diurnal curve in Figure 1 shows absolute cortisol values instead of $\log$ (cortisol) values.

To assess differences in changes of $\log$ (cortisol) levels over time, repeated-measures analyses of variance (MANOVAs) with 'group' (DP + vs DP $-v s \mathrm{NC}$ ) as between subjects factor and 'time' as within-subjects factor were used. Main effects of time and group, as well as interactions between 'time' and 'group' were further analyzed using difference contrast tests. The underlying diurnal cycle (as a measure of overall cortisol production over the day: samples 1, 4, 5, and 6) and the cortisol awakening response (CAR: samples 1-3) were analyzed separately (Edwards et al, 2001).

As measures of the CAR, the area under the curve (AUC), with reference to zero, and area under the response curve (AURC), with reference to the first awakening sample were computed as well. The AUC reflects the mean cortisol secreted within $1 \mathrm{~h}$ after awakening, whereas the AURC reflects the total increase in cortisol secretion from baseline during the first hour after awakening (Edwards et al, 2001).

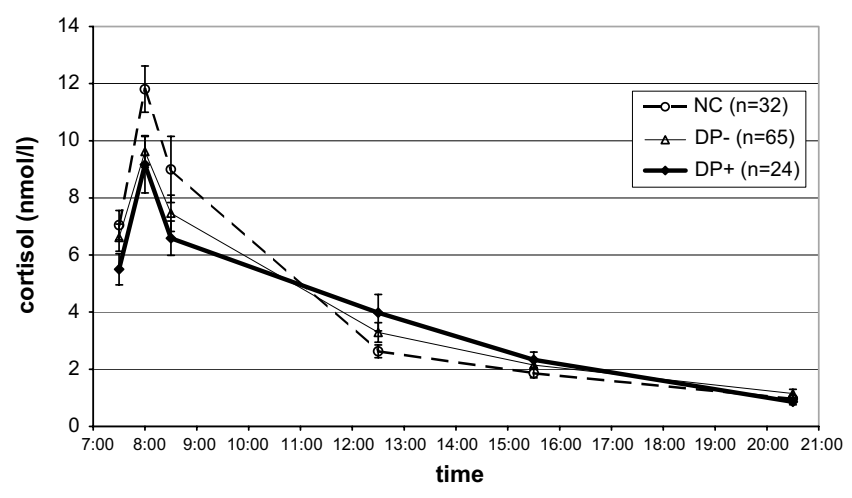

Figure I The response to awakening and the diurnal cycle of cortisol. Means and standard error scores are indicated. NC, normal controls: DP-, diversion project without disruptive behavior disorder: DP +, diversion project with disruptive behavior disorder.

ANOVAs were performed to test for differences between groups (DP + vs DP $-v s \mathrm{NC}$ ) in AUC and AURC.

To assess associations between dimensional measures of disruptive behavior and HPA activity, correlations between the delinquency, aggression, and externalizing scales of the CBCL and the CAR (AUC and AURC) were tested using Spearman correlation tests. All post hoc analyses were corrected with the Bonferroni method for multiple comparisons.

\section{RESULTS}

\section{Behavioral Measures}

A one-factor ANOVA was used to compare scores on the CBCL scales for delinquency and aggression, between the three subgroups (see Table 1). A significant overall group difference was found for both scales (all $p<0.01$ ). Additional post hoc tests also revealed a significant difference between DP + and both $\mathrm{DP}-$ and NC for both scales (all $p<0.01)$. On the delinquency scale, there was a stepwise increase in problems, with a trend toward higher scores in the DP - group as compared with the NC group $(p=0.058)$, and the DP + group scoring significantly higher than the DP- group $(p<0.01)$.

\section{Cortisol Measures}

A graphic representation of the diurnal cortisol cycle for the three subgroups is given in Figure 1. Repeated-measures MANOVA for the diurnal cycle (samples 1, 4, 5, and 6; excluding the CAR) revealed a significant main effect of time $(\mathrm{F}=255.89, p<0.001)$, a significant group by time interaction $(\mathrm{F}=2.34, p<0.05)$, but no main effect of group $(\mathrm{F}=0.29, \quad p=0.75)$. Difference contrast tests showed significant decreases in $\log$ (cortisol) for each sample as compared with the previous samples (all $p<0.001$ ). The significant group by time interaction was mainly attributable to a significant difference between groups in decline between sample one and four $(\mathrm{F}=4.94, p<0.01)$. Post hoc analyses revealed a significant group by time interaction for $\mathrm{DP}+v s \mathrm{NC}(\mathrm{F}=7.63, p<0.001)$, which was mainly attributable to a smaller decline in the DP + group between 
sample 1 and 4. When comparing cortisol levels at individual time points, a significant difference was found at half an hour after awakening $(p<0.05)$, but not at any of the other time points.

A repeated-measures MANOVA, with greenhouse-geisser correction, on the CAR (samples 1-3) revealed a main effect of time $(\mathrm{F}=27.59, p<0.001)$, a main effect of group $(\mathrm{F}=3.24, p<0.05)$, but no interaction between group and time $(\mathrm{F}=0.82, p=0.50)$. Difference contrast tests showed that the main time effect was primarily attributable to an increase in $\log$ (cortisol) between sample 1 and sample 2 $(\mathrm{F}=62.46, p<0.001)$. Employing post hoc tests, a significant group difference was found between the DP + group and the NC group $(p=0.05)$. For the CAR, additional ANOVAs were conducted to assess the effect of group on the AUC and AURC from samples 1-3. For the AUC there was a significant difference between groups $(\mathrm{F}=3.41, p<0.05)$. Post hoc analyses showed a significant smaller AUC in the $\mathrm{DP}+$ than in the NC group $(p<0.05)$. The AURC was not significantly different between groups.

For analyzing the relationship between cortisol and dimensional measures of antisocial behavior, one-tailed Spearman's correlation tests were performed using measures of the CAR (AUC and AURC) and CBCL scores on delinquency and aggression. These revealed an inverse correlation between the AUC and delinquency (Spearman $\rho=-0.18, p<0.05)$, but no other significant correlations.

\section{DISCUSSION}

The aim of this study was to compare the diurnal cycle of cortisol, and the CAR, between male adolescents attending a delinquency diversion program, with or without a diagnosis of disruptive behavior disorder ( $\mathrm{DP}+v s \mathrm{DP}-$ ), and NC. The findings have shown the $\mathrm{DP}+$ group to be different from the NC group with respect to HPA functioning. First, as compared with the NC group, the diurnal cycle of the DP + group was characterized by a slower decrease in cortisol. Second, in the first hour after waking up, although all subgroups showed a similar increase in cortisol form baseline, the DP + group had significantly lower overall cortisol levels than the NC group.

In all subgroups, the expected pattern of cortisol levels during the day was found, characterized by a steep increase during the first $30 \mathrm{~min}$ after awakening and a gradual decrease over the rest of the day. The observed differences between the DP + group and the NC group were related to dynamic aspects of HPA functioning. First, the DP + group showed lower overall cortisol levels during the CAR than the NC group. As noted before the CAR is superimposed on the underlying diurnal pattern, comprising aspects of basal activity as well as dynamic changes during the first hour after awakening (Clow et al, 2004). In healthy populations, the specific finding of low overall salivary cortisol secretion during the CAR has been negatively correlated to longer term stress (Thorn et al, 2006). It was also found in army recruits during an 11-week physical training course (Clow et al, 2006). As such, it may be an indication of an increase in experienced longer term stress in the DP + group. Interestingly, studies investigating children who had gone through stressful experiences have reported similar results, as this current study with respect to low morning cortisol levels (King et al, 2001; Goenjian et al, 1996). Although none of the subjects in the current study had a PTSD diagnosis, other stress-related factors, like early adverse life events and recent stressful events, that could have an effect on HPA axis functioning were not assessed. Future studies on HPA activity and antisocial behavior investigating the role of stressful events in more detail are warranted to study whether such factors could explain the current finding of low cortisol levels during the CAR.

The current data showed no significant differences between groups with respect to cortisol increase from waking up to $30 \mathrm{~min}$ later. However, the smaller overall cortisol output in DP + boys during this period in which cortisol levels normally rise dramatically, resembles results from a study in a similar group showing these boys to have a lower cortisol output in reaction to a laboratory stressor than NC boys (Popma et al, 2006). As such, the current results may reflect low reactivity to stimulation of the HPA axis in DP + boys.

Second, a slower decrease in cortisol levels during the day was observed in the DP + group as compared to the NC group. Even though a steep decline was observed in all groups from half an hour after awakening to one hour after awakening, the decline over the rest of the day was significantly slower in the $\mathrm{DP}+$ group than in the $\mathrm{NC}$ group. Possibly, the normal feedback mechanisms that react to the peak in cortisol levels during the first hour after awakening might have been activated less in the DP+ group because of the low overall levels, resulting in a less robust down regulation of cortisol levels after the morning peak.

Although statistically nonsignificant, by visual inspection the DP- group showed cortisol levels that were mostly in between those of the NC and the DP + group, which may suggest a gradual difference in HPA functioning from the $\mathrm{NC}$ over the $\mathrm{DP}-$ to the $\mathrm{DP}+$ group. This idea may be strengthened by the finding of a gradual increase in CBCL delinquency scores from the NC over the DP-, to the DP + group, and by the finding of a significant, though modest, inverse correlation between CBCL delinquency scores and AUC for cortisol levels in the first hour after awakening.

The finding of low post-awakening cortisol levels in the $\mathrm{DP}+$ subgroup are in line with other studies that have found low cortisol levels in antisocial children (McBurnett et al, 2000; Pajer et al, 2001; Tennes et al, 1986; Tennes and Kreye, 1985). Notably, low cortisol levels were only observed in the first hour after awakening, whereas during the rest of the day the levels were not significantly different between groups, emphasizing the need for repetitive cortisol sampling over the day in future studies. Although some previous studies in antisocial populations sampled cortisol in the morning, their mixed results may still have been caused by not taking into account awakening time. Future studies in groups of juveniles with antisocial behavior problems sampling cortisol with strict reference to awakening time are therefore warranted.

The present results suggest that HPA activity might differentiate boys with a DBD diagnosis, and subsequently a poor prognosis, from boys with less serious antisocial behavior problems and boys with no behavior problems. As mentioned before, HPA functioning has been studied within 
the paradigm of the low arousal theory. First, low arousal, as reflected by low HPA activity, could lead to more sensation seeking (Zuckerman, 1979). Although the current finding of low cortisol levels in the first hour after awakening may be in line with this assumption, this explanation seems to be less plausible because no significant differences in cortisol levels were observed during the rest of the day. Second, a decrease in dynamic and reactivity of the HPA axis could be associated with fearlessness and therefore with low responsiveness to social cues, for example, punishment (Raine, 1993). The current results may support this notion as the $\mathrm{DP}+$ group particularly showed reduced responsiveness of the HPA axis. Mechanisms like the latter might be important in explaining the poor prognosis and the limited effectiveness of current treatments for children with a DBD diagnosis.

This study focused on antisocial behavior defined as having an ODD or CD diagnosis. In the DSM-IV, ODD, and $\mathrm{CD}$ are grouped together as externalizing disorders, together with ADHD. Although there is a high comorbidity for $\mathrm{DBD}$ and $\mathrm{ADHD}$, and both ADHD and DBD children have been found to be at risk for antisocial behavior in adulthood (Mannuzza and Klein, 2000), previous research has shown that the relationship between low cortisol and externalizing behavior problems is specific for ODD/CD children, independent of comorbid ADHD (Snoek et al, 2004).

Some methodological limitations of the present study need to be noted. First, for practical reasons saliva was sampled on 1 day only. Although cortisol levels have a rather high intraindividual stability (Wust et al, 2000), several authors recommended obtaining cortisol samples over two or more consecutive days in order to control for day-to-day variations. However, this is the first study in antisocial children, to our knowledge, that has collected salivary cortisol data at specific time points after awakening, with strict reference to awakening time, and at several time points during the rest of the day.

Second, no electronic monitoring of compliance to the sampling protocol was performed. In recent studies, noncompliance to salivary sampling protocols at home was reported, resulting in blunted cortisol responses to awakening (Kudielka et al, 2003; Broderick et al, 2004). In the current study, a number of precautions were taken to avoid noncompliance, and no evidence was present for differences in compliance between groups.

In spite of these limitations, this study successfully extends to earlier findings on the relationship between HPA activity and antisocial behavior in children. Future studies should continue to establish the role of HPA functioning in antisocial behavior by studying cortisol levels in resting conditions and, in addition, during stressful situations. Furthermore, most studies to date cannot clarify whether alterations in HPA activity are the cause or the consequence of disruptive behavior problems. Interestingly, results from recent studies suggest that low cortisol could be predictive for later disruptive behavior problems (Shoal et al, 2003). The present study does not answer this question, but from the fact that DP- boys showed cortisol values in between DP + and NCs, although not significantly, it could be hypothesized that some boys within the DPgroup have a HPA activity level that is similar to the DP + group and as such might be at greater risk for developing more serious behavioral problems, whereas others might have cortisol levels similar to the NC group and might not be persistent delinquents. Future research should be aimed at elucidating the developmental course and interactions of HPA activity and disruptive behavior problems by conducting longitudinal studies, sampling cortisol repeatedly during the day and using strict cortisol sampling protocols. In order to contribute to this issue, a follow-up study in the current study population will be conducted. A better understanding of the mechanisms involved in the development, persistence and prognosis of disruptive behavior, including a better knowledge of the biological predispositions to antisocial behavior, should ultimately result in earlier and more effective interventions.

\section{ACKNOWLEDGEMENTS}

This study was supported by the Hersenstichting Nederland (Dutch Brain Foundation, Grant no. 9F0.08).

\section{REFERENCES}

Aardal E, Holm AC (1995). Cortisol in saliva-reference ranges and relation to cortisol in serum. Eur J Clin Chem Clin Biochem 33: 927-932.

Aardal-Eriksson E, Karlberg BE, Holm AC (1998). Salivary cortisol-an alternative to serum cortisol determinations in dynamic function tests. Clin Chem Lab Med 36: 215-222.

Achenbach TM (1991). Manual for the Child Behavior Checklist/ 4-18 and 1991 Profile. University of Vermont: Burlingtion, VT.

Azar R, Zoccolillo M, Paquette D, Quiros E, Baltzer F, Tremblay RE (2004). Cortisol levels and conduct disorder in adolescent mothers. J Am Acad Child Adolesc Psychiatry 43: 461-468; discussion 469-472.

Broderick JE, Arnold D, Kudielka BM, Kirschbaum C (2004). Salivary cortisol sampling compliance: comparison of patients and healthy volunteers. Psychoneuroendocrinology 29: 636-650.

Clow A, Edwards S, Owen G, Evans G, Evans P, Hucklebridge F et al (2006). Post-awakening cortisol secretion during basic military training. Int J Psychophysiol 60: 88-94.

Clow A, Thorn L, Evans P, Hucklebridge F (2004). The awakening cortisol response: methodological issues and significance. Stress 7: 29-37.

Coste J, Strauch G, Letrait M, Bertagna X (1994). Reliability of hormonal levels for assessing the hypothalamic-pituitaryadrenocortical system in clinical pharmacology. $\mathrm{Br} J$ Clin Pharmacol 38: 474-479.

Dettling AC, Gunnar MR, Donzella B (1999). Cortisol levels of young children in full-day childcare centers: relations with age and temperament. Psychoneuroendocrinology 24: 519-536.

Edwards S, Evans P, Hucklebridge F, Clow A (2001). Association between time of awakening and diurnal cortisol secretory activity. Psychoneuroendocrinology 26: 613-622.

Gerra G, Zaimovic A, Avanzini P, Chittolini B, Giucastro G, Caccavari $\mathrm{R}$ et al (1997). Neurotransmitter-neuroendocrine responses to experimentally induced aggression in humans: influence of personality variable. Psychiatry Res 66: 33-43.

Goenjian AK, Yehuda R, Pynoos RS, Steinberg AM, Tashjian M, Yang RK et al (1996). Basal cortisol, dexamethasone suppression of cortisol, and MHPG in adolescents after the 1988 earthquake in Armenia. Am J Psychiatry 153: 929-934.

Kerr M, Tremblay RE, Pagani L, Vitaro F (1997). Boys' behavioral inhibition and the risk of later delinquency. Arch Gen Psychiatry 54: $809-816$. 
King JA, Mandansky D, King S, Fletcher KE, Brewer J (2001). Early sexual abuse and low cortisol. Psychiatry Clin Neurosci 55: 71-74.

Kirschbaum C, Hellhammer DH (1994). Salivary cortisol in psychoneuroendocrine research: recent developments and applications. Psychoneuroendocrinology 19: 313-333.

Klimes-Dougan B, Hastings PD, Granger DA, Usher BA, ZahnWaxler C (2001). Adrenocortical activity in at-risk and normally developing adolescents: individual differences in salivary cortisol basal levels, diurnal variation, and responses to social challenges. Dev Psychopathol 13: 695-719.

Kruesi MJ, , Schmidt ME, Donnelly M, Hibbs ED, Hamburger SD (1989). Urinary free cortisol output and disruptive behavior in children. J Am Acad Child Adolesc Psychiatry 28: 441-443.

Kudielka BM, Broderick JE, Kirschbaum C (2003). Compliance with saliva sampling protocols: electronic monitoring reveals invalid cortisol daytime profiles in noncompliant subjects. Psychosom Med 65: 313-319.

Lahey BB, Loeber R, Quay HC, Frick PJ, Grimm J (1992). Oppositional defiant and conduct disorders: issues to be resolved for DSM-IV. J Am Acad Child Adolesc Psychiatry 31: 539-546.

Mannuzza S, Klein RG (2000). Long-term prognosis in attentiondeficit/hyperactivity disorder. Child Adolesc Psychiatr Clin N Am 9: 711-726.

Maughan B, Rutter M (2001). Antisocial children grown up. In: Hill J, Maughan B (eds). Conduct Disorders in Childhood and Adolescence. Cambridge University Press: Cambridge. pp 507-552.

McBurnett K, Lahey BB, Rathouz PJ, Loeber R (2000). Low salivary cortisol and persistent aggression in boys referred for disruptive behavior. Arch Gen Psychiatry 57: 38-43.

Mezzacappa E, Tremblay RE, Kindlon D, Saul JP, Arseneault L, Seguin J et al (1997). Anxiety, antisocial behavior, and heart rate regulation in adolescent males. J Child Psychol Psychiatry 38: 457-469.

Moss HB, Vanyukov MM, Martin CS (1995). Salivary cortisol responses and the risk for substance abuse in prepubertal boys. Biol Psychiatry 38: 547-555.

Pajer K, Gardner W, Rubin RT, Perel J, Neal S (2001). Decreased cortisol levels in adolescent girls with conduct disorder. Arch Gen Psychiatry 58: 297-302.

Popma A, Jansen LMC, Vermeiren R, Steiner H, Raine A, Van Goozen SHM et al (2006). Hypothalamus pituitary adrenal axis and autonomic activity during stress in delinquent male adolescents and controls. Psychoneuroendocrininology 31: 948-957.

Pruessner JC, Wolf OT, Hellhammer DH, Buske-Kirschbaum A, von Auer K, Jobst S et al (1997). Free cortisol levels after awakening: a reliable biological marker for the assessment of adrenocortical activity. Life Sci 61: 2539-2549.

Raine A (1993). The Psychopathology of Crime: Criminal Behavior as a Clinical Disorder. Academic Press, Inc.: San Diego, CA, USA.

Raine A (2002). Biosocial studies of antisocial and violent behavior in children and adults: a review. J Abnorm Child Psychol 30: 311-326.

Scerbo AS, Kolko DJ (1994). Salivary testosterone and cortisol in disruptive children: relationship to aggressive, hyperactive, and internalizing behaviors. J Am Acad Child Adolesc Psychiatry 33: 1174-1184.

Schulz KP, Halperin JM, Newcorn JH, Sharma V, Gabriel S (1997). Plasma cortisol and aggression in boys with ADHD. J Am Acad Child Adolesc Psychiatry 36: 605-609.

Scott S, Knapp M, Henderson J, Maughan B (2001). Financial cost of social exclusion: follow up study of antisocial children into adulthood. BMJ 323: 191.
Shaffer D, Fisher P, Lucas CP, Dulcan MK, Schwab-Stone ME (2000). NIMH Diagnostic Interview Schedule for Children Version IV (NIMH DISC-IV): description, differences from previous versions, and reliability of some common diagnoses. J Am Acad Child Adolesc Psychiatry 39: 28-38.

Shoal GD, Giancola PR, Kirillova GP (2003). Salivary cortisol, personality, and aggressive behavior in adolescent boys: a 5-year longitudinal study. J Am Acad Child Adolesc Psychiatry 42: 1101-1107.

Snoek H, Van Goozen SH, Matthys W, Buitelaar JK, van Engeland $\mathrm{H}$ (2004). Stress responsivity in children with externalizing behavior disorders. Dev Psychopathol 16: 389-406.

Stoff DM, Pasatiempo AP, Yeung J, Cooper TB, Bridger WH, Rabinovich H (1992). Neuroendocrine responses to challenge with dl-fenfluramine and aggression in disruptive behavior disorders of children and adolescents. Psychiatry Res 43: 263-276.

Sulon J, Demey-Ponsart L, Beauduin P, Sodoyez JC (1978). Radioimmunoassay of corticosterone, cortisol and cortisone: their application to human cord and maternal plasma. J Steroid Biochem 9: 671-676.

Susman E, Dorn L, Inoff-Germain G, Nottelmann E, Chrousos G (1997). Cortisol reactivity, distress behavior, and behavioral and psychological problems in young adolescents: a longitudinal perspective. Journal Res Adolesc 7: 81-105.

Tennes K, Kreye M (1985). Children's adrenocortical responses to classroom activities and tests in elementary school. Psychosom Med 47: 451-460.

Tennes K, Kreye M, Avitable N, Wells R (1986). Behavioral correlates of excreted catecholamines and cortisol in secondgrade children. J Am Acad Child Psychiatry 25: 764-770.

Thorn L, Hucklebridge F, Evans P, Clow A (2006). Suspected non-adherence and weekend versus week day differences in the awakening cortisol response. Psychoneuroendocrinology 31: 1009-1018.

Van Goozen SH, Matthys W, Cohen-Kettenis PT, Buitelaar JK, van Engeland $H$ (2000). Hypothalamic-pituitary-adrenal axis and autonomic nervous system activity in disruptive children and matched controls. J Am Acad Child Adolesc Psychiatry 39: 1438-1445.

Van Goozen SH, Matthys W, Cohen-Kettenis PT, Gispen-de Wied C, Wiegant VM, van Engeland H (1998). Salivary cortisol and cardiovascular activity during stress in oppositional-defiant disorder boys and normal controls. Biol Psychiatry 43: 531-539.

Vanyukov MM, Moss HB, Plail JA, Blackson T, Mezzich AC, Tarter RE (1993). Antisocial symptoms in preadolescent boys and in their parents: associations with cortisol. Psychiatry Res 46: 9-17.

Virkkunen M (1985). Urinary free cortisol secretion in habitually violent offenders. Acta Psychiatr Scand 72: 40-44.

Wechsler D (1974). Manual for the Wechsler Intelligence Scale for Children-Revised (WISC-R). Psychological Corporation: New York.

Weizman R, Dick J, Gil-Ad I, Weitz R, Tyano S, Laron Z (1987). Effects of acute and chronic methylphenidate administration on b-endorphin, growth hormone, prolactin and cortisol in children with attention deficit disorder and hyperactivity. Life Scis 40: 2247-2252.

Woodman DD, Hinton JW, O'Neill MT (1978). Cortisol secretion and stress in maximum security hospital patients. J Psychosom Res 22: 133-136.

Wust S, Wolf J, Hellhammer DH, Federenko I, Schommer N, Kirschbaum C (2000). The cortisol awakening responsenormal values and confounds. Noise Health 2: 79-88.

Zuckerman M (1979). Sensation Seeking: Beyond the Optimal Level of Arousal. Lawrence Erlbaum Associates: Hillsdale, NJ, USA. 